\title{
Deutsche Allergologie im Aufwind
}

$\mathrm{B}$ ereits im letzten Sommer widersprachen wir der kritischen Bewertung der deutschsprachigen Allergieforschung durch Bild der Wissenschaft (Heft 3/99), denn ein Aufschwung des internationalen Ansehens war schon damals unverkennbar, u.a. durch die epidemiologische Forschung der vergangenen zehn Jahre.

Innerhalb des vergangenen Jahres haben sich weitere Fortschritte ergeben. Hierzu hat neben der individuellen Aktivität zahlreicher Wissenschaftler v.a. auch die staatliche Förderung der Forschung in der Allergologie und der Pneumologie seit 1998 beigetragen. Ziel der Förderung ist insbesondere die langfristige Etablierung von allergologischen und pneumologischen Strukturen an den Hochschulen, um die Forschung, aber auch die Krankenversorgung und die Lehre auf diesen Gebieten zu stärken.

Klinische Forschergruppen sind derzeit in fünf deutschen Universitätsstädten eingerichtet worden: Berlin, Bochum, Freiburg, Marburg, München. Die allergologischen Themen reichen von der Kontaktallergie (Freiburg) bis zum Asthma bronchiale durch allergische und nicht-aller-

\section{"langfristige Etablierung von aller- gologischen und pneumologischen Strukturen an den Hochschulen"}

der entstandenen Strukturen, insbesondere der neu eingerichteten C3-Professuren, an den Universitäten vorgesehen. Es ist zu hoffen, dass dies nur der Anfang einer verstärkten Präsenz der Allergologie an den Hochschulen ist.

Die verstärkte Forschungsförderung der Allergologie und Pneumologie verdeutlicht, dass die verantwortlichen staatlichen Instanzen zumindest ansatzgische Faktoren (Freiburg, Bochum). Sowohl die genetischen Grundlagen allergischer Erkrankungen (Freiburg, München, Berlin) als auch Zellen und Zytokine (Freiburg, München, Bochum, Marburg) werden bearbeitet. Tiermodelle herrschen vor, um die $\mathrm{Pa}$ thogenese aufzuklären (München, Bochum, Marburg, Berlin). Sowohl den Allergenen (Freiburg, München) als auch viralen Ursachen (Bochum) und Umweltnoxen (München) ist breiter Raum in der Pathogeneseforschung gewidmet. Die Forschungsförderung ist auf insgesamt sechs Jahre ausgelegt. Anschließend ist die feste Etablierung weise erkannt haben, welche Defizite auf diesen Gebieten vorherrschen, trotz der Häufigkeit und der ökonomischen Bedeutung der entsprechenden Erkrankungen. Zu dieser Erkenntnis der Politik haben die zahlreichen Aktivitäten unserer Kollegen aus dem In- und Aus-

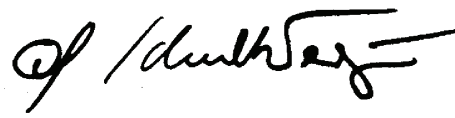

Prof. Dr. G. Schultze-Werninghaus land beigetragen, die beratend in den zuständigen Gremien tätig sind und denen hierfür gedankt sei.

Besonders zu danken ist an dieser Stelle aber Frau Christiane Herzog, die bereits lange vor ihrer Zeit als Gattin des Bundespräsidenten und insbesondere während dieses Lebensabschnitts unermüdlich für die Verbesserung der Versorgung von Patienten mit Atemwegsund Lungenerkrankungen gekämpft hat, keineswegs nur auf dem Gebiet der Mukoviszidose. Sie hat keinen Termin mit den zuständigen Kultusministerien und keine kritische Intervention gescheut, um in Zusammenarbeit mit den Fachgremien eine flächendeckende Versorgung der Bevölkerung sicherzustellen, auch als sie bereits schwer erkrankt war. Ihr Tod am

19. Juni 2000 berührt uns zutiefst.

Die zunehmende Zahl internationaler Publikationen aus dem deutschsprachigen Raum dokumentiert die Fortschritte der deutschen Allergologie. Auch die Vergabe des Kongresses der Europäischen Akademie für Allergie und klinische Immunologie (EAACI) vom 9.-13. Mai 2001 nach Berlin unter der Leitung von Prof. Dr. U. Wahn macht die internationale Wertschätzung deutlich.

Wir wünschen unseren Lesern einen erholsamen Sommer.

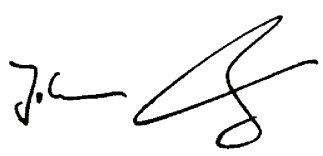

Prof. Dr. Dr. J. Ring 